\title{
The Rules of Engagement: UtilizIng improve to Build Relationship- Centered Care
}

Catherine Braxton

Silver Dawn Training Institute, USA

\author{
"Correspondence author \\ Catherine Braxton \\ Silver Dawn Training Institute \\ USA \\ Phone: 219-649-1732 \\ Facebook : facebook.com/DementiaRAW \\ E-mail : silverdawninfo@gmail.com
}

Submitted : 23 April 2020 ; Published : 8 May 2020

\section{Abstract}

During this unprecedented time of older adults (specifically those living with dementia) requiring services, caregiving and social opportunities amongst their places of residence (including long term care facilities, senior apartment buildings, supportive living spaces and within the community), it is imperative that we adjust our current model of care from "patient-centered" to "relationship-centered".

The most reasonable, practical and cost effective manner to encourage this shift to "relationship-centered care" is a focus on value based communication techniques that can:

Ensure that caregivers/ caretakers continually put the person living with dementia first

Encourage positive experiences during interactions and, Ensure supportive relationships within the future based on trust Several different types of communication interactions and interventions have been attempted and continue to be utilized by caregivers across the board, however they are laden with pit falls that have not addressed or minimized the issue of staff satisfaction, staff turnover, family satisfaction and enjoyment of interactions. Over 20 years ago it was common place to use the concept of "Reality Orientation" which caused caregivers to drag the person living with dementia kicking and screaming into our reality, not allowing them to remain in thiers. Year's later new concepts such as "Therapeutic Fibs" and "Fantasy Validation" became mainstream. Although these ideas were a true growth in resident centric communication they were problematic within their semantics. Family caregivers were unsure what a "Therapeutic Fib" was and if they were comfortable being told to "lie" to their loved ones. Although it was considered therapeutic in nature, many caregivers felt either dishonest or bound by religious doctrine that discouraged such interactions. The same issue held true for "Fantasy Validation". The medical-type jargon itself created difficulty for a family caregiver to understand and therefore implement.
Continuity of care is difficult to maintain when professional caregivers refer to and use such communication techniques yet the family caregivers find it more difficult to implement. With the increased awareness and implementation of Naomi Feil's concepts of utilizing Validation Therapy with persons living with dementia, new growth of communication was seen. However, continued difficulty with semantics, infrequent inclusive training for family caregivers and a lack of instantaneous tools were absent, allowing for the continued frustration in appropriate, honorable and effective communication for all involved. According to Robert L. Canon, LCMW:

Development of a training curriculum and protocol which has the flexibility to meet the needs of diverse caregivers should be a priority.... Content should be Flexible enough for use with all levels of caregivers including family, aids, nurses, Administration, housekeepers and maintenance personal (55).

By introducing the Rules of Improve as the new value based communication strategy, caregivers of all types, professional capabilities and interpersonal relationships can quickly embrace the simplistic terminology, understand the positive outcomes with this relationship centered approach and acquire the tools and techniques immediately for use. Improved was created as a means to encourage relationships to be built amongst people who had nothing in common. At its core, it encourages the building of something out of nothing. At its roots, the rules of improve were used with underserved populations of youth that came from various backgrounds. With varied spoken language becoming an increasing barrier to create relationships, improved rules and exercises were created to break that barrier and encourage "partnershipcentered" interactions that ensured positive experiences for all as well as desires of future relationships based on trust built during the exercises. 
Although there are many rules of improved, there are four main rules that can be easily

Understood and implemented by any person, from any age.

These four rules are:

1. Yes, And...,

2. Relinquish Your Agenda,

3. Make Your Partner Look Good, and

4. The Gifts.

These four rules fully embrace, recognize and enforce the truth that older adults should be viewed as embodying inherent worth. Naomi Feil stated, "They are seen as having wisdom of their years, being worthy of help in resolving issues of loss and deserving support of their dignity and worth" (1992).

By embracing the concepts of The Rules of improved as a foundation for value based communication and "relationship centered care" for those persons living with dementia, we can 1. Bridge the generation gaps that currently increase solitude and isolation among the older adult.

\section{Address staff job satisfaction and turnover}

Empower family caregivers with a method of caregiving that does not compete with any personal agenda and provides immediate tools for use, establish positive, honorable and validating experiences for the person living with dementia and, secure the opportunities for future relationships and communication connections based on trust. In the field of dementia care there have been many evolutions to appropriate and effective communication strategies over the years. There have been numerous changes to the long term care industry as a whole however the one thing that remains lacking is a cost effective, easily implemented "Gold Standard" in communication training that is practical in learning and application and can appeal to all types of caregivers regardless of age or education.

According to the authors of "The facilitators of communication with people in dementia care setting: an interview study with healthcare workers", current staff training is lacking in many aspects and specifically enhanced communication training is necessary, needing to be "more practical in learning methods and applications and ... support the transfer of training into practice." [1-4].

Family caregivers continue to struggle with "how to communicate" with their loved ones. The reason for this is multifaceted. There are very few opportunities for appropriate communication training for the adult-child and their family so novice caregivers resort to "elderspeak" (defined as childlike communication efforts). According to Kristine M. Williams, "An increased probability of Resistiveness to care occurred with elderspeak $(.55,95 \% \mathrm{Crl}$, and 44-.66), compared with normal talk (.26, 95\% Crl,. .12-.44-.66. Communication training has been shown to reduce elderspeak and may reduce Resistiveness to care..."
Secondly, any training that is provided by direct care staff (typically informal) is laden with technical jargon that creates disconnect and confusion. According to the Asian Nursing Research study conducted by Jing-Jy Wang, "The results... serve as a reference for planning dementia communication education...to enhance...communication abilities...to increase nurses' patient-centered communication abilities with the ultimate goal of improving quality of care.

Finally, communication techniques and theories vary among professional caregivers, specifically direct care staff and upper management. When a lack of continuity in communication is displayed in a long term care facility it is difficult to adhere to a standard of care.

An existing communication model that was created for this exact purpose: connecting those who may have difficulty understanding each other is called improved. At its core, The Rules of engagement. The Rules of improved avoid elderspeak and provide a "Gold Standard" in practical, applicable, honorable and cost effective communication training that can be easily implemented, understood by all family members as well as professional care staff and provide for a continuity improved is built upon the concept of unconditional acceptance and a positive volley among communicating partners.

The 4 Rules of improved that provide this "Gold Standard":

1. Yes, And...,

2. Make Your Partner Look Good,

3. Relinquish Your Agenda and,

4. The Gifts compliment the concepts in Validation Therapy as described by Naomi Feil, "The basic needs of the client for love and acceptance are the basis for the therapeutic relationship and are fostered.

The Rules of Engagement: Utilizing improved to Build Relationship-Centered Care

The Rules of improved were originally designed to create connections among underserved youth originating from various nationalities and socio-economic backgrounds. Grouping together several young adolescents who do not speak the same language could lead to difficulties in communication and relationship building, if left to each individual's agenda without the vision of a shared goal. The rules of improved allow for connection in even the most tense of moments. Improved was not originally designed as a means to laughter and comedy but rather based on rules of engagement that build something out of nothing.

In recent years improved has been solely viewed as a form of comedy. Comedian groups, television programs and local theater troupes perform and present improved as a stage act to elicit laughter from the audience. Underneath the humor is a set of rules that the players must abide by in order to establish relationships that move a scene forward. Four specific rules of improved: 1. Yes, And Relinquish Your Agenda Make Your Partner Look Good. 
The Gifts have shown to be highly effective in communicating with persons living with dementia. While bringing the initial and intended concept of improved full circle, the rules can be used with staff, family members and clients who originate from various nationalities, socio-economic backgrounds, cognitive and language abilities.

When a long term care facility fully embraces these four rules of improved in their daily engagement with each other, their clients and the family members they work with, a dramatic decrease in stress, anxiety, fear and problematic behaviors become evident for all. The focus shifts from the rules of grammar and proper speech patterns, the "what" in communication, to the rules of engagement: the "how" and the "why" in communication. Emphasis on "How" to communicate translates to specific language used (verbal and physical) that allows all partners to look good during the interaction. The "Why" in communication translates to an intentional (rather than impulsive) focus on the main objective, essentially, what are we looking to achieve here, in the long run.

This state of the art concept of introducing these rules of improved to long term care staff can be easily implemented, applied, practiced and immediately utilized. The rules of improved do not require extensive reading, lectures, seminar trainings or webinars. Additionally the rules of improve allow for the staff to practice daily with anyone, anywhere. This builds muscle memory in its application, allowing for it to be a skill that is not "turned on and turned off" when entering the workplace, but rather a lifelong competence.

In the current atmosphere of long term care where staff turnover is at an all-time high and quality of care varies depending on staffing, budgets and funding, it is time for a critical change to take place in the industry. Family members are looking for the best placement for their loved ones, while balancing guilt, shame, indecisiveness and cost. The decision to utilize long term care is a difficult one. If families are forced to choose a facility solely based on cost, they may be reeling with the possibility of lower quality of care. This should not be the case. The rules of improved and engagement allow for exceptional interaction and relationship-centered care, regardless of budgetary restrictions.

Furthermore, the application of improve rules during engagement do not require extensive, if any, additional knowledge of disease processes and diagnosis. In fact, they are such groundbreaking concepts that focus solely on moment to moment relationship building and partnership pairing, it becomes irrelevant what a persons' diagnosis or prognosis is. It is in-the-moment, errorless, positive interaction that allows for all participants to bond, even if for the smallest amount of time. When working with persons living with dementia, this becomes even more vital as the varying levels of cognitive ability differ with each client.
The four rules of improve should be embraced by all levels of the care team, from the top down, as daily modeling and application of its effectiveness will only be as strong as the team that fully embraces its power. When family members witness the shift in engagement by everyone, they themselves will be impacted by the quality of care delivered by the team.

Polished marble floors and winding staircases do not translate to superb levels of care. When a facility cannot afford the latest and greatest in technological advances, this does not mean that their ability to deliver exceptional relationship-centered care should suffer. There is no "one quick fix" to the uphill battle we face within our industry. To restore caregiving back into a rewarding experience for all, we must consider concepts in communication and connection that are accessible to all players of the caregiving team. It is only then that the prospect of long term care will enhance the lives of those we care for and for ourselves as well.

As we take a deeper dive into the 4 rules of improve that can greatly improve communication, connection and engagement among caregivers, those they care for and their co-care partners, we will dissect how each of the rules can be easily understood and implemented by everyone: "Yes, and"- As the primary rule of improve, is used by all novel and seasoned improve artists, it is the most vital component of connection. By saying "Yes" we are simply being in agreement. By saying "And..." we are adding to the dialogue. "Yes, and..." can be used in a variety of interactions including a verbal exchange, a physical expression and on an emotional level.

At its most basic form, using "Yes, And..." in active verbal dialogue keeps the conversation moving in a forward motion. "Yes" communicates to the resident that what they are saying is unconditionally accepted. "And..." conveys that the listener is interested. Consider the alternative of "No." This is demeaning, shame producing and can create a total shut down in connection. "Yes" is not the only word that can be used in this scenario, any positive affirmation of understanding can be used such as "What a good idea" or "What an interesting way to think about that", or "Wow, tell me more".

"And" is the intentional choice to move the connection further. It creates an equal dialogue among two people rather than a power struggle of teacher and student. When we add to the conversation with a simple question or comment about what we are hearing, we are communicating that we care, we understand, we want to know more. This type of affirming banter among a person living with dementia can be emotionally uplifting and encouraging.

An example of the verbal "Yes, and..." interaction between a person living with dementia (PLD) and a caregiver may sound like this: PLD "Do you see the purple bird in the tree?" Caregiver: "Yes, I do see something moving in that tree. I wonder if it is a bluebird. Did you have bluebirds where you lived?"

PLD: "When I moved to the Midwest with my husband I saw so many more birds than I did in New York. We even made bird 
houses in the summer."

Caregiver: "I love watching birds gather to a bird house. Did you know we have an aviary here? I can take you to see some of the birds we have."

Using the "Yes, And..." approach on a physical level requires the listener to hear and understand their bodies. It is considering that more than $50 \%$ of communication is done via facial expressions, body language and tone of voice. With this in mind, it opens space for connections to be made with people that are progressively losing their ability to communicate verbally or are currently nonverbal. With the understanding that the majority of body language is universal, it is not difficult to deduce, by examining and mirroring body language and facial expressions, "What" a person living with dementia may be feeling at the moment. Just because a person can no longer speak in a manner that we can comprehend does not mean that we cannot find, within ourselves, the ability to understand them. This is the heart of what improved was built upon.

If a person living with dementia is sitting alone, wringing their hands, head down, and squinting their eyes we can deduce that that person feels alone, scared, in pain or anxious. By approaching these possibilities in mind we can match the person where they are. This is the "Yes" portion of being in agreement physically. We would choose not to approach like a cheerleader with overwhelming energy, but rather a mirror of their own body. By doing this we channel the bio feedback loop within ourselves that gives us information as to why we might ever hold our bodies in that manner. Then the approach is full of empathy, understanding and pairing in the form of matching. It is two equals sharing a moment, even an unpleasant one, and that is what creates connection.

When we can be in agreement with someone emotionally, we can tap into the most vital component of "Yes, and..." With a combination of verbalizations, tone, inflections and the reading of body language, we can get to the heart of what is being communicated emotionally by the person. Although their language may not clearly describe what they are feeling, we can ask ourselves "What is this person really communicating?" and then connect with the idea that "I have been there too." The misconception that neuro-typical caregivers cannot relate to the emotions that a person living with dementia is erroneous. If we as caregivers take a moment to look, listen, and truly pay attention to the entire message, we can tap into our deepest empathic abilities and understand what the person is feeling at the moment. It is the feeling that we agree with. It is the feeling that we "Yes": "Wow, It looks like you are having a very hard time. I have been there too. I want to support you. Can you tell me more?"

This technique may even include some self disclosure on the caregiver's part. Consider sharing with a PLD that you have recently felt anxious when you were lost. Sharing that you were scared and alone will create a connection that you truly do understand how the PLD may be feeling. It is a connection that will reveal ways in which you can help further.
Due to the fact that improved was created for youth to learn, understand and immediately utilize makes this a great tool for any and all caregivers. With a simple two or three minute tutorial on what "Yes, and..." means (to be in agreement no matter what, and then to add something to it), even a novice caregiver can absorb and use the technique. Additionally the simplicity of the language "Yes, and..." allows for easier comprehension, compared to the current terminology used such as "therapeutic fibs" and "fantasy validation". When a family caregiver is encouraged to use these techniques, they often get caught up in the definition of "therapeutic", "fib", and "validation". By eliminating the confusing jargon and the time it takes to explain such techniques, the family caregiver can quickly gain insight and a useful tool to create connection immediately. Furthermore the term "fib" can create a disconnect among families that do not subscribe to being deceitful towards the PLD. The term "fantasy" in "fantasy validation" suggests that the PLD is delusional and cannot be affirmed or trusted. The language of this technique itself can perpetuate shameful interactions.

The second rule of improved that enhances connection is called Relinquish Your Agenda. At its essence, it is the ability to be truly in the moment, regardless of what is going on around you. On the improved stage a player will join his troupe midscene. If the player enters with his own agenda, or his own expectations, then the entire troupe is thrown off kilter. The player must enter with an empty head, an empty agenda, and then find his place among the scene with his partners.

In caregiving the agendas that often create miscommunication and lack of connection include the agenda to be right and the desire to give advice. These two predominant agendas create disconnect, frustration on both parties and ultimately breakdown in the relationship. Many novice caregivers feel that they must correct the PLD when they communicate in ways we do not understand or seem "meaningless". Consider this dialogue:

PLD: "I need to get to work"

Novice Caregiver: "You don't work anymore"

PLD: "What do you mean, how I am going to pay the mortgage" Novice Caregiver: "You don't need to pay a mortgage, you don't have a house anymore."

PLD: "No house, Oh my goodness, what will we do? What will my husband say?" Novice Caregiver: "Don't worry about your husband. He passed away 5 years ago."

Notably this conversation created anxiety, fear, shame and shock to the PLD but it also created a downward spiral for the novice caregiver. No one was getting anywhere fast except frustrated and exasperated, leaving no room to create a connection that would lead to a trusting relationship in the future. As the novice caregiver held tightly to the agenda that they were right and the PLD was wrong, a true disconnect was inevitable. What was required here was the empty space that the caregiver could create in which they would listen to understand, not listen to fix. 
An additional agenda that is prevalent in conversations is the "desire to give advice". Many caregivers travel this slippery slope without even realizing it, especially when they employ such tactics as distraction and redirection. Offering advice instead of listening to what is really being said, the emotion behind the words, can also lead to discouraging interactions between caregivers. Consider a situation in which a caregiver tells a PLD "not to worry about it" or "it's not a big deal, let's just go to the bingo game instead." Essentially, the advice that the caregiver is providing can make the PLD feel unheard, unimportant and dismissed.

Advice creates a divide among people, a hierarchy of right and wrong, an atmosphere of teacher and student. This inequity in conversation may lead to misunderstandings, frustration, shame and a feeling of inadequacy on the part of the novice caregiver. When family members don't feel that they can be successful at communicating with their loved one, they are less likely to visit and provide that much needed familiar interaction. If a seasoned caregiver were to relinquish their agenda of giving advice and instead meet the novice caregiver where they are, the interaction may be more impactful. Consider this scenario where the seasoned caregiver is in the moment, utilizing their full empathy to create a connection with the family member:

Seasoned Caregiver: "That had to be so difficult for you to see your mom struggle like that. When I started working with her she was so adamant about going to work, I didn't know what to say to her that would calm her down."

Family Member: “I know. I can't seem to get it through to her that she lives here now. I keep telling her that this is her home but she fights me constantly."

Seasoned Caregiver: "She fought me too. We struggled for some time as well. It got to the point where she wouldn't even take my assistance to the dining room. I felt so awful about it" Family Member: "What did you do? You are so great with her. She loves seeing you. I just don't understand why she is so angry with me"

Reasoned Caregiver: "I guess I let go of my need to be right. If she felt like she needed to go to work, I talked to her about work. If she felt like she had to catch the bus, then we talked about bus schedules and her first trip on a bus. I stopped fighting against where she thought she was, I just joined her there."

His act of Relinquishing Your Agenda is a practice in mindfulness and intentionality. It requires the caregiver to let go of their own attachments of right and wrong, and listen with empathy for the emotional message being conveyed.

The third applicable rule of improved within the caregiving setting is called Make Your Partner Look Good. Within an improved troupe, when each player is focused on making their partners look good, giving them the limelight, then ultimately all partners look good. If one partner chooses to dominate the scene, the energy changes and the audience can feel the tension. Improved itself is a practice of give and take.
In the caregiving scene it can be powerful to intentionally make the care-receiver (the PLD) look good regardless of the scenario. The goal is to never put the PLD in shame or cause them to do the heavy lifting during an opportunity to connect. Asking questions, drilling for memories, making statements such as "Don't you remember..." and "I'm Sorry" does nothing to make our partner look good (or feel good). It elicits stress, conflict and often shut-down. When we work to make our partners look good, we expedite connection.

When we say we are sorry as a response to a PLD expressing a difficult moment, we break down the ability to connect. From a psychological perspective, the words "I'm Sorry" fill an uncomfortable space and put us (as caregivers) in a neutral space while placing the PLD in a negative space. It requires them to do the heavy lifting in the conversation, the responsibility lies in them to rectify the imbalance. "Trust isn't rebuilt with apologies. Trust is restored when we become more trustworthy and that is best accomplished when we take steps to change."- Veronica Monet As quoted in the YourTango.com article 'Raise Your Hand If You're Tired of Saying You're Sorry.' by Veronica Monet 'Empathy is much more valuable to your partner than your guilt. We all want to feel truly heard and deeply understood.

Guilt, however, runs rampant among caregivers as they watch their loved ones or daily residents succumb to dementia and display deterioration of skills. Expressing this guilt is difficult to resist, however the alternative to expressing sadness and guilt is to be specifically grateful in time of unrest. This practice is intentional, deliberate and purposeful. By fighting against the urge to say "I'm Sorry "when working with a PLD, and instead, taking a moment to reflect on how to express being specifically grateful, (such as saying "Thank you for sharing that with me"), a caregiver displays recognition in the honor of sharing. The caregiver builds trust by communicating that they appreciate that they were the ones chosen to share in a difficult moment.

Additionally, Making Our Partners Look Good requires contemplative awareness. In essence there are hundreds of different ways to respond to a difficult situation, and it is in the words that we choose, the tone that we use and the intention behind both that can determine if we make our partners look and feel good, or not. Consider the situation in which a PLD has difficulty eating with a spoon without dripping soup on their shirt. A caregiver could approach and reprimand, approach and take the spoon away, or even approach and offer a "bib". Or a caregiver could approach and offer the PLD a "dining scarf" in an effort to "keep that beautiful shirt looking so wonderful. "This approach makes our partner look and feel good. In turn they may show gratitude, appreciation and trust, which makes the caregiver look and feel good too.

A very meaningful use of Make Your Partner Look Good is the adoption of the method among caregivers themselves. Not only should direct care staff keep the mantra of "Make Your Partner Look Good "when working with each other during 
times of conflict with a PLD, but additionally management staff would elevate direct care staff by using the technique as well. Consider the positive consequences in staff determination if an administrator of a building, while talking to a PLD, stopped a nursing assistant, pulled him/ her to the side and said: "I am embracing my failure with Mrs. Johnson right now. I am just not communicating with her in an effective manner. You are so great with Mrs. Johnson, you seem to always know the right thing to say. Would you mind stepping in? I do not want to upset her further."

The Administrator looks good because they are being vulnerable to one of their mistakes. The nursing assistant looks good, and feels good, because they are being called in as the hero in the situation, able to handle it better than their superior. And ultimately Mrs. Johnson looks good because a negative experience with the Administrator was halted and a well suited assist was provided to de-escalate any potential frustrations.

The fourth rule of improved that can greatly enhance communication and connection is called "The Gifts". During an improved scene, players will look for hints (gifts) given by their other players in order to move the scene forward. It may be a word, a gesture or a physical movement. Players can take that gift as a jumping off point to alter direction of the scene while keeping all players involved. The same is true in caregiving. If caregivers look at all elements of an interaction or expression they will find gifts that will help in the process of connection. By looking and listening for key elements conveyed in dialogue, verbal and non-verbal expressions, caregivers can create peak moments of success and reminiscing.

Furthermore, in improve, failure is celebrated. This is not a typical response to mistakes and mishaps in caregiving, however if caregivers begin to look at mistakes as a place of growth, learning and future improvement, then missteps can be viewed as gifts in themselves. This concept, when adopted from the top down, can dramatically alter staff morale and vulnerability to the pitfalls of caregiving rather than the current climate of burnout, frustration, and evasion of truth during conflict. When a member of upper management can admit their mistakes and create an environment that embraces a "round table discussion" of those mistakes, then other levels of caregivers will see and follow this model, creating a workplace that thrives on transparency and the focus of growth within caregiving.

An improve game called Take That Back is essential in helping members of an improve troupe try on the concepts of different approaches to the same scenario. When caregivers embrace Take That Back in day to day interactions with PLD, they not only adopt the "fail beautifully "concept, but they put it into practice by "Taking Back "their initial reaction or response and open themselves up to try a new approach with the PLD. When working with individuals who live with memory impairments, it can be advantageous for the caregiver to leverage the potential "short loop "and make an attempt to try again. As long as the initial response/ reaction embodied good intentions, it is possible that the PLD will remember the positive attempt and emotion related to that and not the actual words.

Consider this dialogue again, in which the caregiver embraces the gift in conversation:

PLD: "Do you see the purple bird in the tree?"

Caregiver: "Yes, I do see something moving in that tree. I wonder if it is a bluebird. Did you have bluebirds where you lived?"

PLD: "No, it is not a bluebird. Can't you see that purple animal! I see it right there." (Gifts: Bluebird, tree, frustration that caregiver did not respond in the way they needed)

(Caregiver now using Take That Back Method)

Caregiver: "Oh, yes, the purple thing moving in the branches. Now I see what you are talking about. I must have overlooked that. I have never seen anything like that. I only had robins where I grew up."

PLD: "Oh, we had all types of birds in Florida. Some were so big they would block the putting green when I would golf."(Caregiver can now shift into positive remenising connection)

By utilizing the concepts of "the gifts", a caregiver can embody the idea of being in agreement by utilizing "Yes, And..." a reminder to "Relinquish their Agendas", and focus solely on "Making their Partner Look good."

The Rules of improved provide a Gold Standard of empathic, respectful and effective communication training for anyone looking to connect with a person living with dementia. Furthermore it is a cost effective manner of training that allows for all types of caregivers to remain educated, involved, provide continuity and connection. The Rules of improve can be practiced anywhere with anyone. This unique dynamic of improve communication allows for the communication techniques to become a lifestyle rather than just a work skill that is turned on and turned off at work. When an individual commits to practicing just one of the rules for an entire week with everyone they come in contact with, they will essentially master the nuances of all four rules within a month. This encourages a strengthening of the improve communication muscle which in turn strengthens the availability of the skill when working with persons living with dementia. Essentially it becomes second nature.

Additionally, the practice of the improve Rules with people other than those living with dementia works to strengthen relationships among caregivers themselves, specifically family members who are often providing different levels of care and contact based on location and availability. Family disagreements that arise from agendas, judgmental viewpoints and non-empathic listening can be reduced by the utilization of these techniques. Again, a strong indicator that the Rules of improved the lives of those living with dementia (as family struggles can be reduced). Finally the improve Rules can be easily practiced in long term care settings weekly with staff 
during "stand-up" gatherings. In addition to weekly update meetings, improve games can be utilized to enhance team building, improve skill sets, allow for a judgment free space to embrace failures beautifully and encourage learning from these "Gifts" rather than exacerbating shame, guilt and staff burn out.

\section{References}

1. Canon, Robert L. LMSW (1995) "The effects of Validation Therapy Training on Satisfaction with communication and quality of relationships between staff and family caregivers and dementia residents in long term care." Institute for Quality Improvement on Long Term Health Care. School of Health Professionals Southwest Texas State University San Marcos Texas. IQILTHC Series Report 95-3. September 1995.

2. Stanyon, Miriam Ruth (2016) "The facilitators of communication with people with dementia in a care setting: an interview study with healthcare workers." Age and Ageing 45(1): 164-170. https://doi.org110.1093/ ageing/afv161. January 2016.

3. Wang, Jing-Jy PhD. "Long-term Care Nurses' Communication Difficulties with people living with dementia in Taiwan." Asian Nursing Research 7(3): 99103. https://doi.org/10.1016/j.anr.2013.06.001 . Access date September 2013.

4. Williams, Kristine N RN (2009) "Elderspeak Communication: Impact on Dementia Care". American Journal of Alzheimer's disease \& Other Dementias 24(1): 11-20. https://doi.org/10.1177/1533317508318472. Access date February 2009.

Copyright: (C2020 Catherine Braxton. This is an open-access article distributed under the terms of the Creative Commons Attribution License, which permits unrestricted use, distribution, and reproduction in any medium, provided the original author and source are credited. 